\section{MS46-O5 Atomic structure of nanoalloy catalysts by resonant high-energy XRD and atomic PDFs analysis}

\section{Valeri Petkov ${ }^{1}$}

1. Dept. Physics, Central Michigan University, Mt. Pleasant, MI 48859 USA

email: petko1vg@cmich.edu

With current technology moving rapidly toward smaller scales nanometer-size alloys are being produced in increasing numbers and explored for various applications, in particular catalytic ones. To understand better and so gain more control over the performance of nanoalloy catalysts, precise knowledge of their atomic-scale structure is needed. With bulk alloys such knowledge is almost straightforward to obtain by Bragg X-ray diffraction (XRD). Unfortunately, Bragg XRD is inapplicable to nanoalloys since their XRD patterns show a limited number of distinct Bragg peaks, if any, and a very pronounced diffuse component. The problem can be solved by employing a non-traditional approach involving high-energy XRD and atomic pair distribution functions (PDF)s analysis [1]. However, for a nanoalloy comprising $n$ atomic species a single XRD experiment yields a total atomic PDF which is a weighted sum of $n(n+1) / 2$ partial PDFs. This could make the interpretation of PDF data for nanoalloys ambiguous. Using resonant high-energy XRD allows particular partial PDFs to be highlighted and others dimmed thus giving very much needed chemical specificity [2-4]. In the talk we will briefly introduce resonant high-energy XRD as applied to atomic PDFs analysis and give examples from several recent studies on nanoalloy catalysts of $\mathrm{Au}-\mathrm{Pt}, \mathrm{Au}-\mathrm{Cu}, \mathrm{Pt}-\mathrm{Pd}$ and $\mathrm{Pt}-\mathrm{Ru}$ families, including building 3D structure models on the basis of total and partial atomic PDFs and using the models for rational consideration of nanoalloy structure-catalytic properties relationships.

[1]. V. Petkov, Materials Today 11 (2008) 28.

[2]. V. Petkov and S. Shastri, Phys. Rev. B 81 (2010) 165428.

[3]. V. Petkov et al., Nano Lett. 12 (2012) 4289.

[4]. V. Petkov et al., J. Phys. Chem. C 117 (2013) 22131. (a)
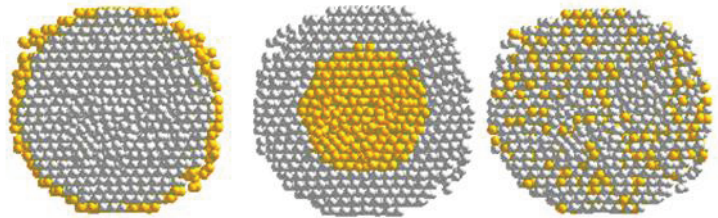

(b)
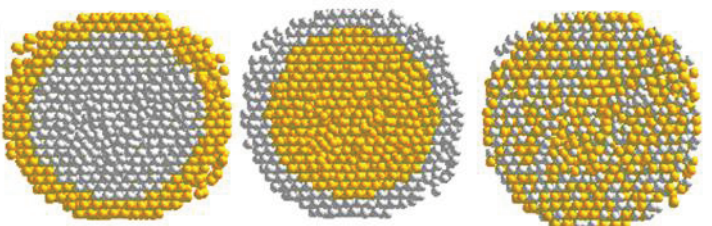

(c)
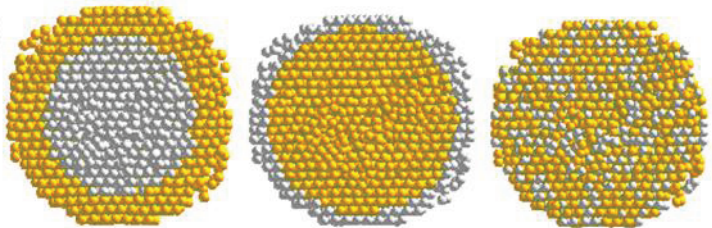

Pt core-Au shell

Au core-Pt shell

Pt-Au alloy

Figure 1. 3D structure models of $5 \mathrm{~nm}$ Au-Pt nanoaloy particles tested and refined against resonant high-energy XRD data as explained in ref. [3].

Keywords: Nanoalloys high-energy resonant XRD atomic PDfs analysis 3D structure modeling 